\title{
AN ELECTRON MICROSCOPIC ANALYSIS OF THE TRANS-SYNAPTIC EFFECTS OF PERIPHERAL NERVE INJURY SUBSEQUENT TO TOOTH PULP EXTIRPATIONS ON NEURONS IN LAMINAE I AND II OF THE MEDULLARY DORSAL HORN
}

\author{
STEPHEN GOBEL \\ Neurocytology and Experimental Anatomy Section, Neurobiology and Anesthesiology Branch, National Institute of Dental \\ Research, National Institutes of Health, Bethesda, Maryland 20205
}

Received September 9, 1983; Revised January 17, 1984; Accepted February 28, 1984

\begin{abstract}
To examine the effects of peripheral nerve injury on second-order neurons in laminae I and II of the medullary dorsal horn, tooth pulps of all mandibular teeth in adult cats on one side were extirpated. This procedure severed and removed the receptors and terminal branches of the primary trigeminal neurons which innervate the tooth pulps of these teeth. The empty pulp chambers were then filled with dental cement to prevent regeneration. At 30 and 60 days postoperatively, membrane-lined cavities had formed inside many of the small-caliber dendrites of second-order neurons in laminae I and II. Cavity formation occurred mainly in dendritic shafts less than $2 \mu \mathrm{m}$ in diameter and involved dendrites with synaptic vesicles as well as those without synaptic vesicles. The cavities extensively hollowed out these dendrites, often occupying more than half the cross-sectional diameter of the shafts and extending for appreciable distances in the long axis of the shaft. The process of cavitation ultimately resulted in the destruction of the affected dendrites. Many cavities became patent to the intercellular space with the cavity membrane establishing continuity with the dendritic membrane. Many cavities often formed in a single dendrite, and such severely cavitated dendrites became reduced to a trabeculated shell which ultimately fragmented into several small pieces. The presence of synaptic connections from a number of different kinds of axonal endings, including scalloped and dome-shaped endings, was not sufficient to prevent cavitation. The actual severing of synaptic connections on the cavitated dendrite appeared to be a relatively late event in the process since small pieces of dendritic debris could still be found clinging to their axodendritic synapses. Evidence that dendrites were being lost from the neuropil was most readily apparent in many of the disrupted glomeruli in lamina II in which many of the scalloped depressions in the central axonal endings that normally contained small dendrites were empty. Many central axonal endings remained in synaptic contact with only a single dendrite which often showed signs of cavitation. Such central endings showed only subtle remaining traces of their normal scalloped contours. This study demonstrates that injury to the distal branches of primary trigeminal neurons which innervate tooth pulps resulted in trans-synaptic degenerative changes in the dendritic arbors of second-order neurons which destroyed finecaliber higher order dendrites.
\end{abstract}

This study's purpose is to try to gain some insight into what happens to second-order neurons in the dorsal horn when they have been deprived of their normal peripheral inputs. The study was designed to look at a situation in which distal processes of primary neurons in peripheral nerves are injured as opposed to a situation in which central processes of primary neurons in either trigeminal or dorsal roots are injured. There are two reasons for acquiring informtion about the effects of such injuries. First, recent new evidence suggests that in distal process injuries the primary neurons survive and maintain their axonal arbors in the dorsal horn in normal topographic position (Sugimoto and Gobel, 1982). Second, injuries to distal processes of primary neurons are more common clinically than injuries to central processes, and these may underlie some chronic pain states, such as trigeminal neuralgia, various phantom limb sensations, and causalgias.
The experiments in this report were designed to examine a situation in which primary trigeminal neurons innervating tooth pulps are injured. Tooth pulp primary neurons comprise an interesting group of primary neurons in that they have a wide range of cell body sizes (Arvidsson and Gobel, 1981) and a wide spectrum of conduction velocities spanning $C$, A-delta, and A-beta ranges (Greenwood et al., 1972; Dostrovsky et al., 1981; Cadden et al., 1983), and yet all have free or unencapsulated nerve endings (Frank, 1966; Harris and Griffin, 1968; Corpron and Avery, 1973; Byers et al., 1982). Tooth pulps are probably one of the most densely innervated structures in the body. For example, even the relatively small incisors of the cat can be innervated by as many as 394 axons (Fried and Hilderbrand, 1981). The tooth pulp system is well known, i.e., we know precisely where the primary axons of tooth pulp neurons terminate centrally in the trigeminal brainstem sensory nuclei 


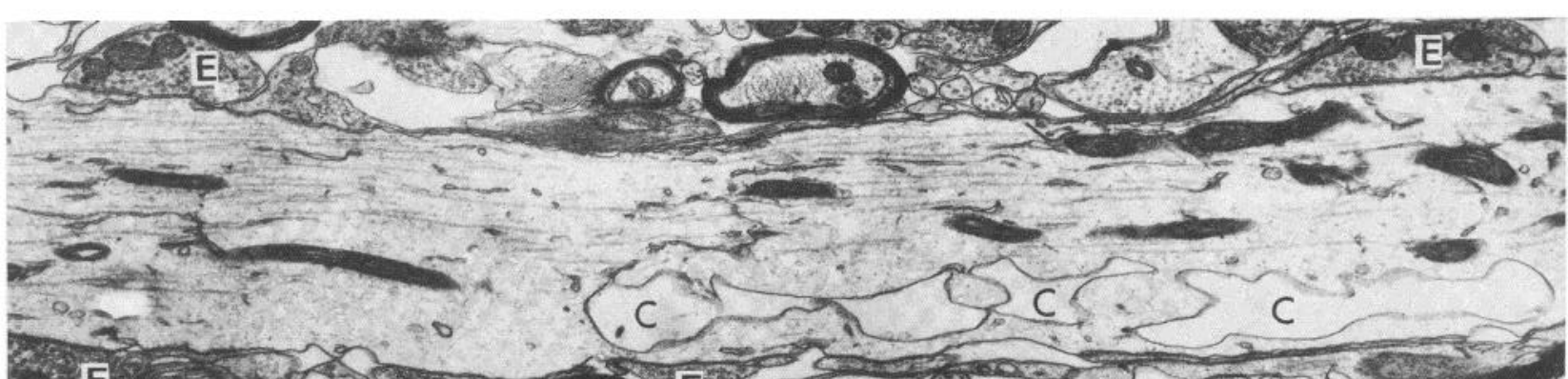

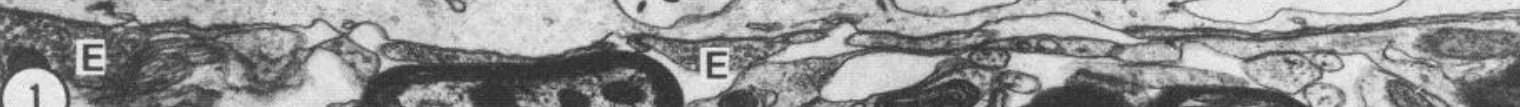

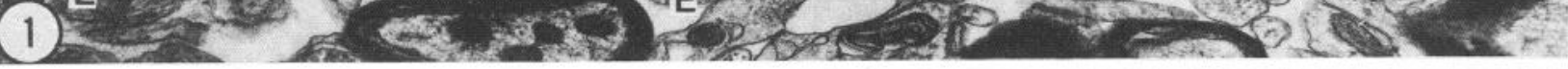

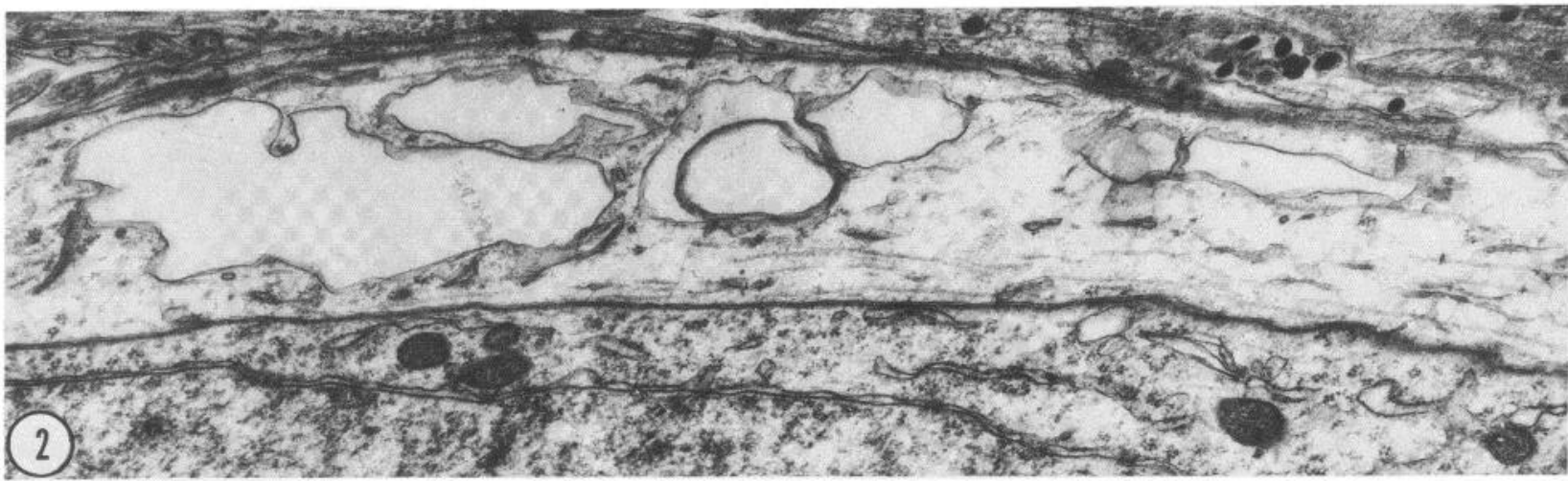

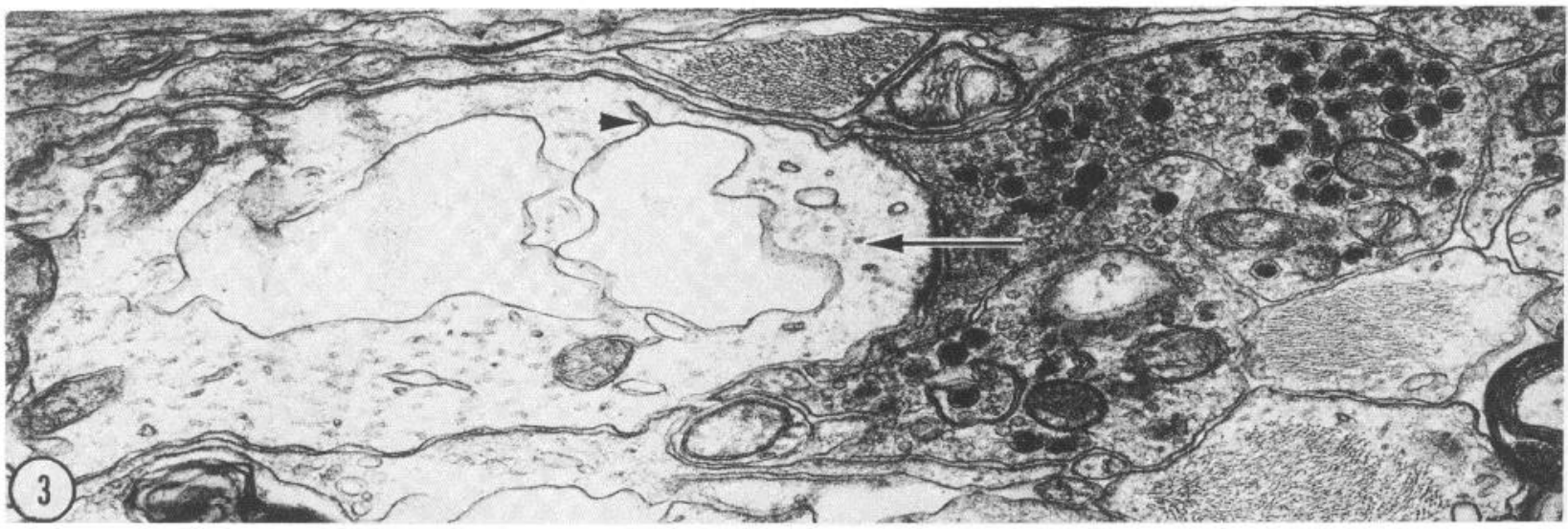

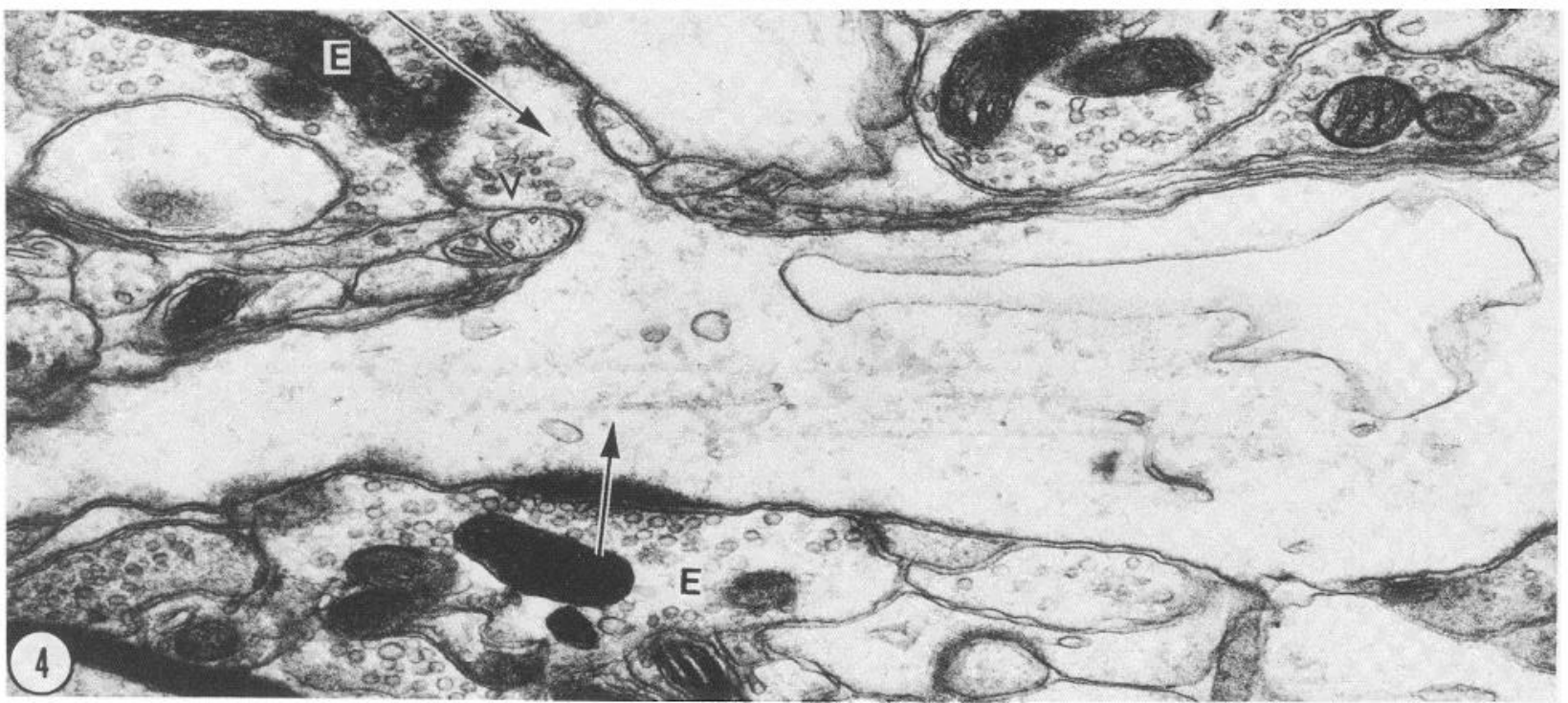


(Arvidsson and Gobel, 1981; Westrum et al., 1981). The system is clinically relevant because tooth pulp primary neurons are frequently injured as a result of dental disease in which pulpal tissue or entire teeth are often destroyed so that the injured primary neurons cannot regenerate back into their normal peripheral receptive fields.

Recent studies using the technique of transganglionic transport of horseradish peroxidase have precisely defined a central termination field of primary trigeminal neurons innervating tooth pulps in laminae I and II of the rostral half of the medullary dorsal horn (Arvidsson and Gobel, 1981). The present study describes a series of degenerative changes which take place inside of many of the small-caliber dendrites of secondorder neurons within the termination field following the severing of the distal branches of tooth pulp primary neurons consequent to pulpotomy. During the first 60 postoperative days, numerous small cavities develop inside of the dendrites, which ultimately lead to their destruction and disappearance from the neuropil of laminae I and II.

\section{Materials and Methods}

Following anesthesia with ketamine, the contents of the pulp chambers of all of the mandibular teeth on one side were removed from four adult cats. The contents of the root canals were not disturbed to avoid any possible damage to the nerve endings in the periodontal ligament. The empty pulp chambers were then sealed with a dental cement. Following postoperative survival times of $14,30,60$, and 90 days the cats were perfused with an aldehyde solution containing a $0.12 \mathrm{M}$ phosphate buffer, $1 \%$ glutaraldehyde, $1 \%$ paraformaldehyde, and $0.2 \%$ $\mathrm{CaCl}_{2}$. Blocks containing the medullary dorsal horn (Gobel et al., 1981b) were then osmicated in a solution containing a $0.14 \mathrm{M}$ phosphate buffer, $8.5 \%$ dextrose, $0.2 \% \mathrm{CaCl}_{2}$, and $1 \% \mathrm{OsO}_{4}$. The specimens were then block stained in uranyl acetate, dehydrated in a series of ethyl alcohols, and embedded in an epoxy resin. Laminae I and II of the dorsomedial third of the ipsilateral medullary dorsal horn which are known to receive the central axonal arbors of primary neurons which innervate tooth pulps (Arvidsson and Gobel, 1981; Westrum et al., 1981) were examined with the electron microscope in transverse and parasagittal planes.

\section{Observations}

In the 2 months following the disruption of primary inputs from the pulpotomized teeth, many of the dendrites of neurons in laminae I and II are being lost from the neuropil. They undergo a series of regressive changes which involve the formation of cavities inside of the shafts of the dendrites. The process of cavity formation takes place mainly inside of smallcaliber dendrites less than $2 \mu \mathrm{m}$ in diameter. 'The cavities usually extend for appreciable distances in the rostrocaudal axis of the dendrite (Figs. 1 and 2) and may often occupy more than half the cross-sectional area of the affected shaft (Fig. 3). Rarely a cavity will distend a dendritic shaft giving it a beaded appearance (Fig. 5), although usually the developing cavities do not disturb the smooth cylindrical shape of the dendrite (Figs. 1 to 3 ). The process of cavitation must involve the dendrites of more than one cell type since cavities are found in dendrites in laminae I, IIa, and IIb and since they also affect dendrites which contain synaptic vesicles (Fig. 4) as well as those that do not.
The developing cavities have an irregular shape. They usually have a main chamber with one or more finger-like recesses which can be quite long (Fig. 4). Many of the developing cavities appear to be joined to flattened saccules of the dendritic agranular reticulum (Figs. 3 and 6). Most of the cavities appear empty, but some contain an amorphous flocculent substance (Fig. 5).

Cavities develop inside of dendrites which receive symmetrical and asymmetrical synapses from many different kinds of axonal endings. These include several different kinds of domeshaped endings (Figs. 6 and 7) and scalloped endings (Fig. 16), including some with many dense-core vesicles (Fig. 3). Synaptic connections can be found on the shafts (e.g., Figs. 6 and 7) as well as on the spine heads (Fig. 4) of cavitating dendrites. Cavities can be found in a dendritic shaft either quite close to the site of a synapse or somewhat removed from it (compare Figs. 6 and 7 ).

Many of the dendritic cavities are open to the intercellular space. At these sites the cavity membrane is continuous with the cell membrane. Cavity openings occur in many $1-$ to $2-\mu \mathrm{m}$ dendritic shafts (Figs. 8 and 9) as well as in some extremely small dendritic processes less than $0.5 \mu \mathrm{m}$ in diameter, some of which may possibly be spine heads (Fig. 11). Cavity openings can be quile narrow (Fig. 8) or extremely wide (Figs. 9 and 10). These openings are found along segments of dendritic shaft that receive synapses from several different kinds of axonal endings (Fig. 8 to 11 ), and they occur in dendrites inside of (Fig. 9) as well as outside of glomeruli (Fig. 9). Cavity openings are not associated with any particular kind of opposing process and have been found opposite glial processes (Figs. 8 and 12a), unmyelinated axons (Fig. 9), and axonal endings (Fig. 10).

The process of cavitation has a destructive effect on the afflicted dendrites. Many dendrites have several cavities forming in them simultaneously, and two or more of these cavities may be open to the intercellular space at the same time (Fig. $12, a$ and $b$ ). These patent cavities give the afflicted dendrites a corrugated appearance and leave several small irregular projections protruding from the main portion of the dendritic shaft (Figs. $12 a$ and $b$ and 13). Ultimately, the process of cavitation leads to the destruction and disappearance of many dendrites from the neuropil of laminae I and II. Figure 14 shows a severely cavitated dendrite in which only a small fragment of the shaft remains. A large space and two nearby pieces of debris attest to the lost portion of the dendritic shaft. Judging by the small remaining cavity in the top of the fragment and the thin fingerlike processes extending from the bottom of the fragment, this dendritic shaft is still in the process of involuting. Extremely small irregular-shaped fragments of dendrites which have undergone severe cavitation are also found in the neuropil still maintaining their synaptic connections with different kinds of axonal endings (Fig. 15).

In addition to the many irregular areas of intercellular space in the neuropil (Figs. 12 to 15), an examination of the glomeruli in lamina II also reveals that many dendrites are being lost from the neuropil. Glomeruli in this lamina typically consist of a centrally situated scalloped axonal ending surrounded by several small dendritic spines and shafts, some of which contain synaptic vesicles (Gobel, 1974, 1976). These dendrites lie im-

Figure 1. Lamina IIa at 60 days. A fine caliber dendritic shaft, $1.6 \mu \mathrm{m}$ in diameter, contains three elongated irregular cavities $(C)$. Four small dome-shaped axonal endings $(E)$ abut the dendritic shaft. Magnification $\times 15,600$.

Figure 2. Lamina IIa at 30 days. Several large cavities which extend for $6.5 \mu \mathrm{m}$ have extensively hollowed out this fine dendritic shaft Magnification $\times 21,800$.

Figure 3. Lamina I at 60 days. A large scalloped axonal ending containing numerous dense-cored vesicles forms an asymmetrical synapse (arrow) on a dendritic shaft which contains two large cavities. One cavity appears to be connected to a cisterna of the agranular reticulum at the arrowhead. Magnification $\times 31,000$.

Figure 4. Lamina IIa at 30 days. A dendrite contains synaptic vesicles $(V)$ in a spine head and an elongated cavity in its shaft. Axonal endings (E) synapse (arrows) on the spine head and shaft. Magnification $\times 30,700$ 


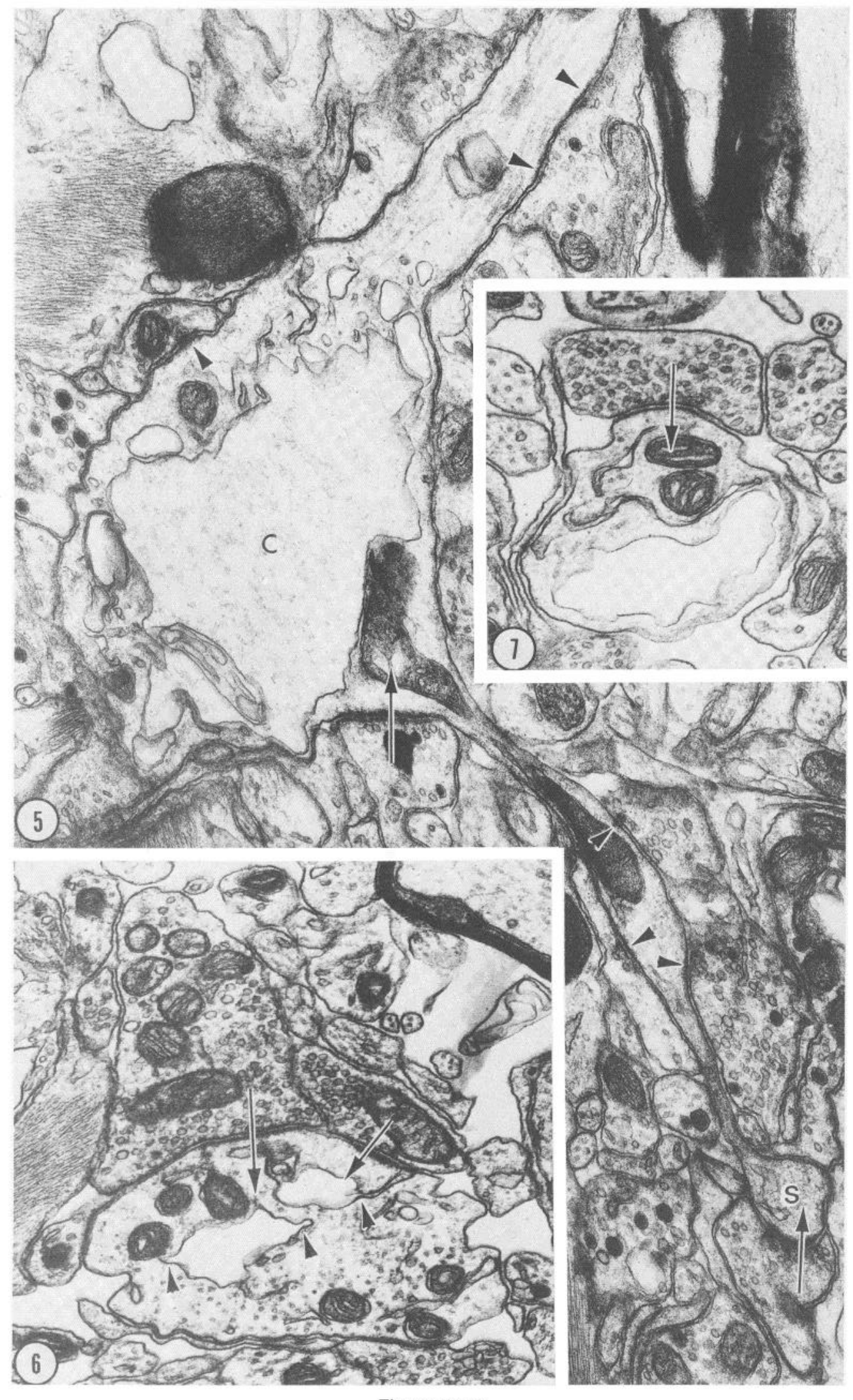

Figures 5 to 7 

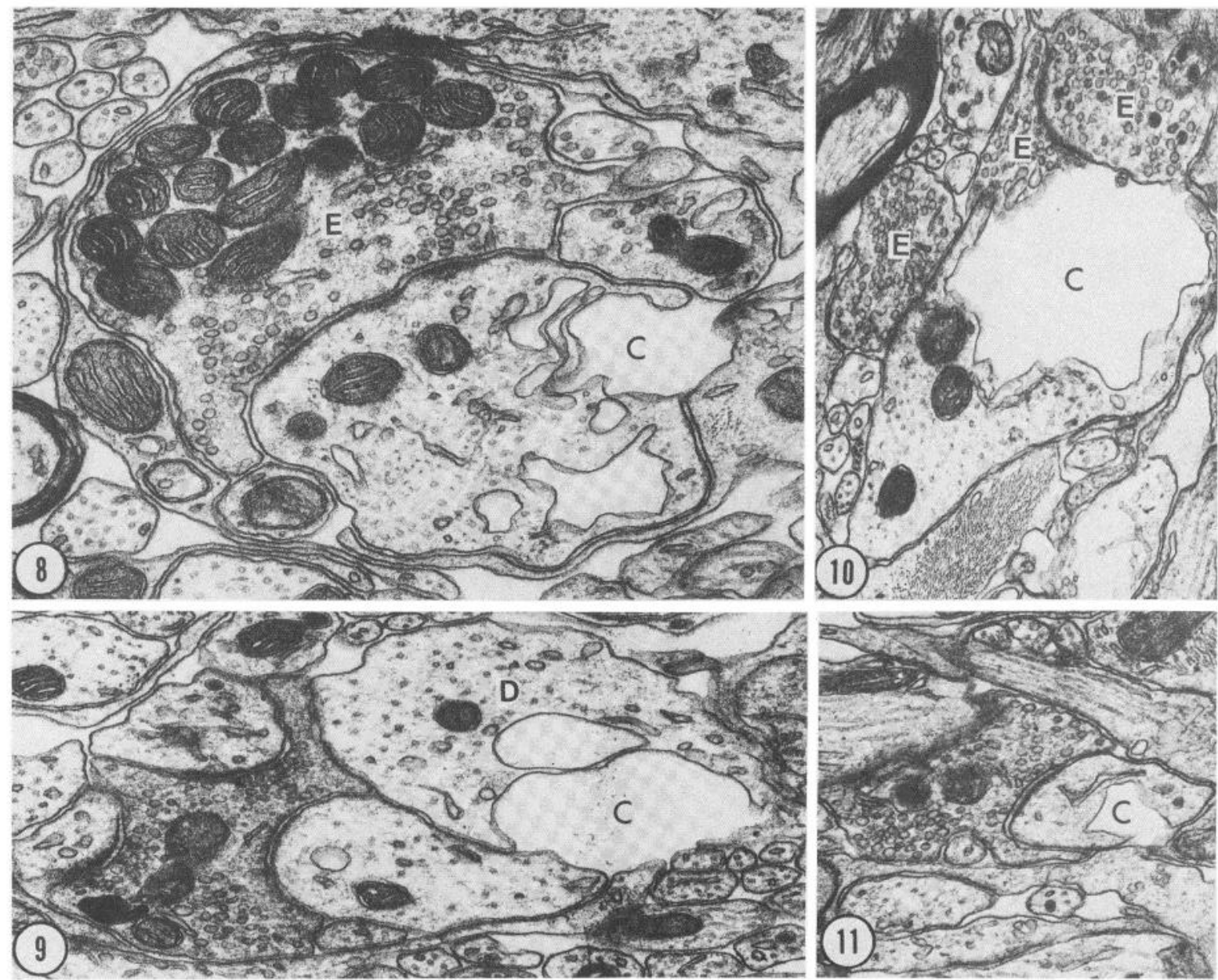

Figure 8. Lamina IIb at 60 days. A small cavity $(C)$ in this dendritic shaft is opened to the intercellular space. Several other small cavities are forming in the dendrite. An axonal ending $(E)$ with relatively few synaptic vesicles forms two synapses on the dendrite. Magnification $\times 29,000$.

Figure 9. Lamina IIa at 60 days. In this glomerulus, one of the dendritic shafts $(D)$ contains a large patent cavity $(C)$ and an adjacent smaller one. Magnification $\times 30,000$.

Figure 10. Lamina I at 60 days. A large patent cavity $(C)$ has eroded about half the cross-sectional area of this dendritic shaft which lies outside of a glomerulus and is surrounded by three small axonal endings $(E)$. Magnification $\times 27,700$.

Figure 11. Lamina I at 60 days. A small dendritic process, possibly a dendritic spine head, contains a patent cavity $(C)$ and receives an asymmetrical synapse from a scalloped axonal ending. Magnification $\times 31,000$.

pressed in the scalloped depressions of the central endings where they receive axodendritic synapses from the central ending and occasionally from other small axons which enter the glomeruli. Many dendrites in the glomeruli contain cavities (Figs. 16 and 18). Other dendrites in the glomeruli are retracting from the surface of the scalloped central ending, and their open cavities leave long, thin, finger-like processes (Fig. 17) which are indicative of open cavities. The actual loss of dendrites from the glomeruli is revealed by empty scalloped depressions in the central axonal ending, such as those in Figure 16. Another indication of the loss of dendrites and disruption of the glomeruli is revealed in Figure 18 in which only a single dendrite remains connected to the central axonal ending of the glomerulus. In many of these disrupted glomeruli only faint suggestions of the scalloped contours of the central endings remain (Fig. 17). Appreciable portions of the surface of these endings are loosely covered by thin glial processes (Figs. 17 and 18), while other portions remain uncharacteristically exposed to the intercellular space.

\section{Discussion}

Recent anatomical studies using the method of transganglionic transport of horseradish peroxidase have shown that laminae I and II of the rostral half of the medullary dorsal horn

Figure 5. Lamina IIa at 30 days. A fine dendrite with a bead-like enlargement gives rise to a long-necked spine $(S)$ in the lower right corner. The bead-like enlargement is extensively hollowed out by a single large cavity $(C)$ and several smaller ones. The large cavity contains amorphous flocculent material. Asymmetrical synapses (arrows) occur on the spine head and bead. Several other structures, presumed to be axonal endings, with only a few synaptic vesicles are also attached to this cavitated dendrite by synapse-like junctions (arrowheads). Magnification $\times 28,400$.

Figure 6. Lamina IIa at 60 days. Adjacent dome-shaped endings form symmetrical and asymmetrical synapses (arrows) on a dendritic shaft which contains two cavities close to the synaptic junctions. Both cavities show tail-like extensions (arrowheads) which suggest connections with the agranular reticulum. Magnification $\times 36,000$.

Figure 7. Lamina IIa at 60 days. The entire bottom half of this dendrite has been destroyed by an extensive cavity, while the upper half of the dendrite, which contains two mitochondria and membranous cisternae, remains intact. A small dome-shaped ending with a mixture of small oval and flattened vesicles forms a symmetrical synapse $($ arrow) on the dendrite. Magnification $\times 39,400$. 

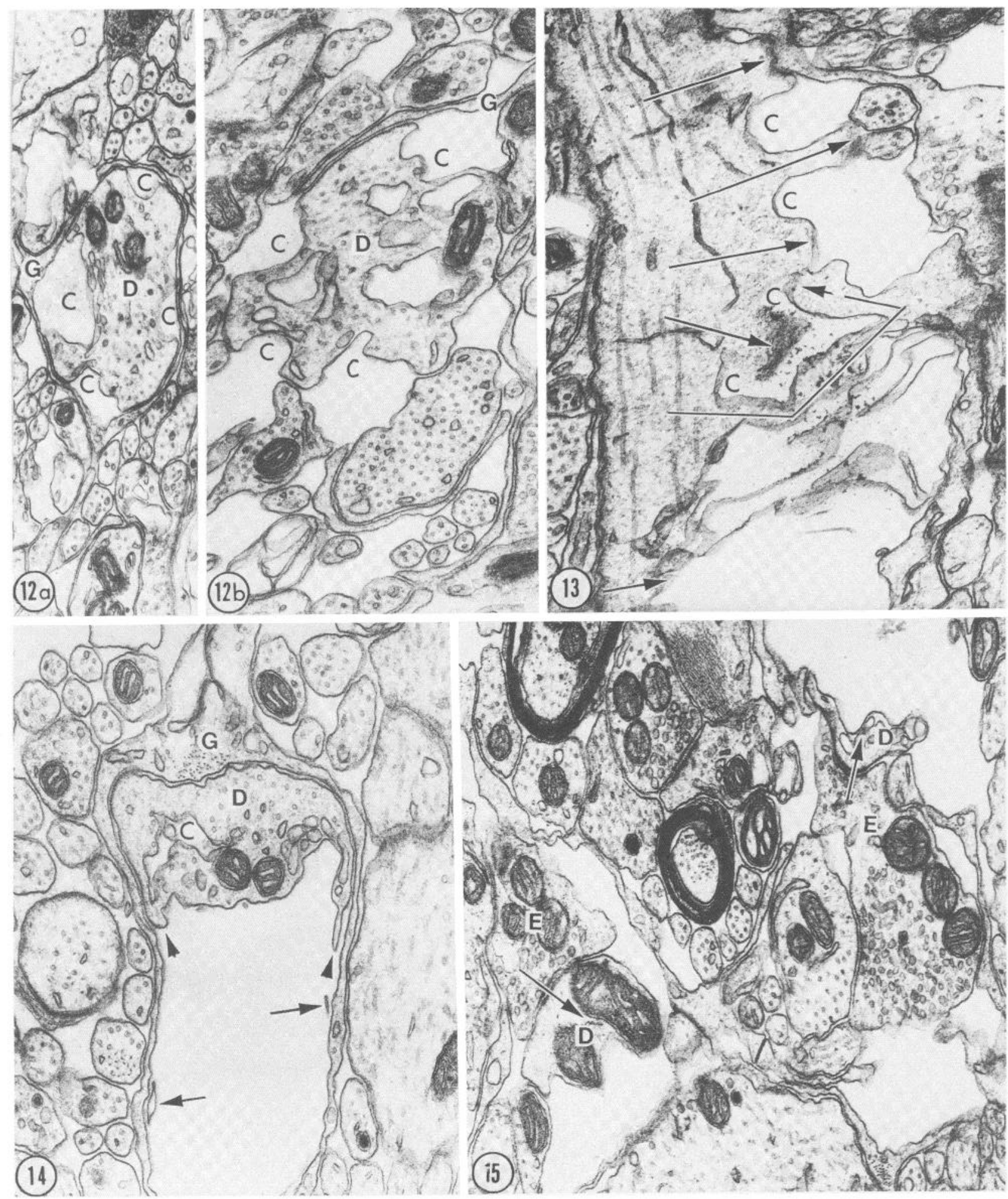

Figure 12. $a$ and $b$, Two examples of transversely sectioned dendritic shafts $(D)$ which show the effects of having had several cavities $(C)$ open to the intercellular space. The patent cavities have altered the smooth, circular outline of the shafts to an irregular corrugated one. In $a$ the cavitated dendrite is completely enclosed in a thin glial process $(G)$, while the one in $b$ is only partially covered by a glial process $(G)$ and lies adjacent to other neural processes. Several small cavities in $b$ continue to erode the dendrite. $a$, Lamina IIa at 60 days. Magnification $\times 27,000$. $b$, Lamina II at 60 days. Magnification $\times 36,400$.

Figure 13. Lamina IIb at 60 days. The fluted surface on the right side of this longitudinally sectioned dendritic shaft shows the destructive effects of severe cavitation on the dendrite. The many irregular extensions (arrows) denote the sites of patent cavities $(C)$. Magnification $\times$ 32,900 .

Figure 14. Lamina IIa at 60 days. Two finger-like processes (arrowheads) project from the remnant of this severely cavitated dendritic shaft $(D)$. Two fragments (arrows) and the large intercellular space within the glial process $(G)$ probably remain from the lost portion of the dendrite. A small cavity $(C)$ continues to erode the remaining portion of the dendritic shaft. Magnification $\times 31,600$.

Figure 15. Lamina IIa at 60 days. The severing of synaptic connections between cavitating dendrites and axonal endings must be a relatively late event. Two small irregularly shaped fragments of severely cavitated dendrites $(D)$ are linked synaptically $($ arrows $)$ to small axonal endings $(E)$. The adjacent enlarged intercellular spaces reflect the lost portions of the dendrites. Magnification $\times 34,200$. 
are major termination sites of primary trigeminal neurons which innervate tooth pulps (Arvidsson and Gobel, 1981; Westrum et al., 1981). In an earlier examination of these laminae and the overlying spinal $V$ tract a number of regressive axonal changes were detected during the first 60 days following tooth pulp extirpation (Gobel and Binck, 1977). For example, some myelinated axons appeared somewhat shriveled, while others contained blackened mitochondria or had disruptions in their myelin sheaths. Some axonal endings resembling primary endings showed a darkening of their axoplasm or lost some of their synaptic vesicles. Similar kinds of intra-axonal changes have been described in axonal endings in lamina II of the spinal dorsal horn following spinal nerve injury (Knyihar and Csillik, 1976). Despite these changes, a more thorough analysis of laminae I and II in the present study has indicated that there is no large-scale Wallerian-like axonal disintegration taking place. The small amount of debris previously reported in lamina I in the earlier study (Gobel and Binck, 1977) has turned out to be an extremely rare occurrence. It is concluded that most of the injured primary trigeminal tooth pulp neurons, despite the possibility of some internal changes in some of their axons, survive the injury as do primary spinal neurons following spinal nerve injuries (Sugimoto and Gobel, 1982).

The major effect of peripheral nerve injuries consequent to tooth pulp extirpations on the CNS neurons of laminae I and II is damage and destruction of many of their small-caliber dendritic branches. Although some dendrites darken and others lose some of their organelles (Gobel and Binck, 1977), the most prevalent form of dendritic destruction is through the process of cavitation. This process is an insidious one, and the afflicted dendrites produce very little debris as they disintegrate. Cavities appear to develop at multiple foci within a dendritic shaft (Figs. 1 and 2). They erode the dendrites as they enlarge and ultimately become connected to the intercellular space (Figs. 8 to 12). The afflicted dendrites are destined to become a trabeculated shell of their original form (Figs. 3 and 12 to 14).

There are several reasons for linking these dendritic changes to the effects of the peripheral nerve injury and not considering them artifacts of the preparative methods. (1) The methods used in this study have been applied over an extended period of time, and cavitated dendrites have never been observed in normal adult animals. (2) Cavitated dendrites have not been seen following a number of other experimental procedures which have damaged inputs to laminae I and II using either identical or very similar preparative methods. These include trigeminal rhizotomies with relatively short-term postoperative survival times, i.e., 3 days and 6 days (Gobel, 1974), our own long-term dorsal root rhizotomies, i.e., 30 days (S. Gobel, unpublished observations) as well as those of others (Ralston and Ralston, 1979), and injury to descending serotonergic inputs using the serotonin neurotoxin 5,6-dihydroxytryptamine (Ruda and Gobel, 1980). (3) Within the tooth pulp extirpation experiments, the prevalence of cavitated dendrites varies at different postoperative survival times. While they are common at 30 and 60 days, they are relatively rare at 14 days and cannot be found at 90 days. (4) At 30 and 60 days, the ipsilateral and contralateral sides look somewhat different. The ipsilateral side contains many cavitated dendrites with some showing patent cavities and loss of portions of the affected dendritic shafts. Cavitated dendrites are only rarely encountered on the contralateral side, and patent cavities have not been seen. The findings of some cavitated dendrites on the contralateral side rules out considering the contralateral side as a possible control. However, it should be added that parallel experiments in which a spinal nerve, i.e., the superficial radial nerve, was transected provide an additional control on the preparative methods. Utilizing the method of transganglionic transport of horseradish peroxidase, the injured primary neurons were found to terminate ipsilat- erally in the cervical $\left(\mathrm{C}_{6}\right.$ to $\left.\mathrm{C}_{8}\right)$ dorsal horn (Sugimoto and Gobel, 1982). Utilizing the same preparative methods as in the tooth pulp extirpation experiments, an electron microscopical analysis of the ipsilateral cervical and lumbar dorsal horns shows many cavitated dendrites in the cervical dorsal horn at 30 and 60 days postoperatively but no cavitated dendrites in the lumbar dorsal horn in the same animals (Sugimoto and Gobel, 1984). (5) Cavitation does not affect all different sized dendrites. Only small-caliber higher order dendrites, i.e., those less than $2 \mu \mathrm{m}$ in diameter, are affected. The large-caliber more basal dendrites are apparently unaffected by the nerve injury. (6) Within the cavitated dendrites, microtubules and other organelles, such as mitochondria, synaptic vesicles (Fig. 4), and membranous components of the agranular reticulum (Fig. 13), are well preserved. In severely cavitated dendrites, the remaining pieces of dendritic shafts also look well preserved, i.e., their microtubules and organelles appear intact (Figs. 12b and 14). (7) The process by which small dendrites are involuting involves a whole series of events which includes the formation of small cavities, enlargement of the cavities, establishment of patency, fragmentation, and frank loss of dendrites from the scalloped depressions of glomeruli (Figs. 16 to 18) as well as from the neuropil outside of the glomeruli (Fig. 14). This entire sequence of events cannot simply be ascribed to deficiencies in the preparative methods. (8) The remaining components of the neuropil surrounding the disintegrating dendrites both inside of the glomeruli (Figs. 16 to 18) and outside of the glomeruli (Figs. 14 and 15) look well preserved. These include several different kinds of axonal endings, unmyelinated axons, myelinated axons, and astrocytic processes.

The source of the cavity membrane is not clear. There are two possible sources. It may arise directly from the agranular reticulum that is normally found in dendritic shafts. Certainly many connections exist between the cavities and small cisternae of dendritic agranular reticulum (Figs. 3 and 6). However, it also may arise from internalization of the cell membrane. What is clear is that ultimately the cavitated dendrites disintegrate and are lost from the neuropil. The many disrupted glomeruli (Figs. 16 to 18), bits of dendritic processes (Fig. 14), some of which are still attached at axodendritic synapses (Fig. 15), and empty intercellular spaces (Fig. 16) stand as evidence to the destructive nature of the cavitation process. Ultimately as the cavitating dendrites disintegrate, synaptic connections are broken as exemplified, for example, by the empty scalloped depressions in the glomeruli in Figure 16. However, the actual breaking of the synaptic junctions must be a relatively late event in the sequence since severely cavitated dendrites are still seen with their synaptic connections intact (Figs. 3, 5, 11, and 15). No isolated postsynaptic densities indicative of broken synapses, such as those seen by Westrum and Black (1971) in subnucleus interpolaris following retrogasserian rhizotomy, were seen in laminae I and II in this study.

The injury to primary trigeminal neurons appears to be a critical factor in the destructive cavitation process. Injury to spinal peripheral nerves also results in cavitation and destruction of small-caliber dendrites in the spinal dorsal horn (Sugimoto and Gobel, 1984). Synaptic inputs to the small-caliber dendrites from a number of different kinds of nonprimary axons, for example, from small dome-shaped endings (Figs. 6 and 7) which include descending serotonergic axons that are known to terminate in these laminae (Ruda and Gobel, 1980), are not sufficient to prevent cavitation.

Several observations have suggested that injured primary neurons survive and maintain their central axonal arbors intact. For example, most axonal endings, including the scalloped endings in the glomeruli in laminae I and II, appear healthy, and, in the case of injury to primary spinal neurons, it has been possible to show that at least some injured primary neurons 

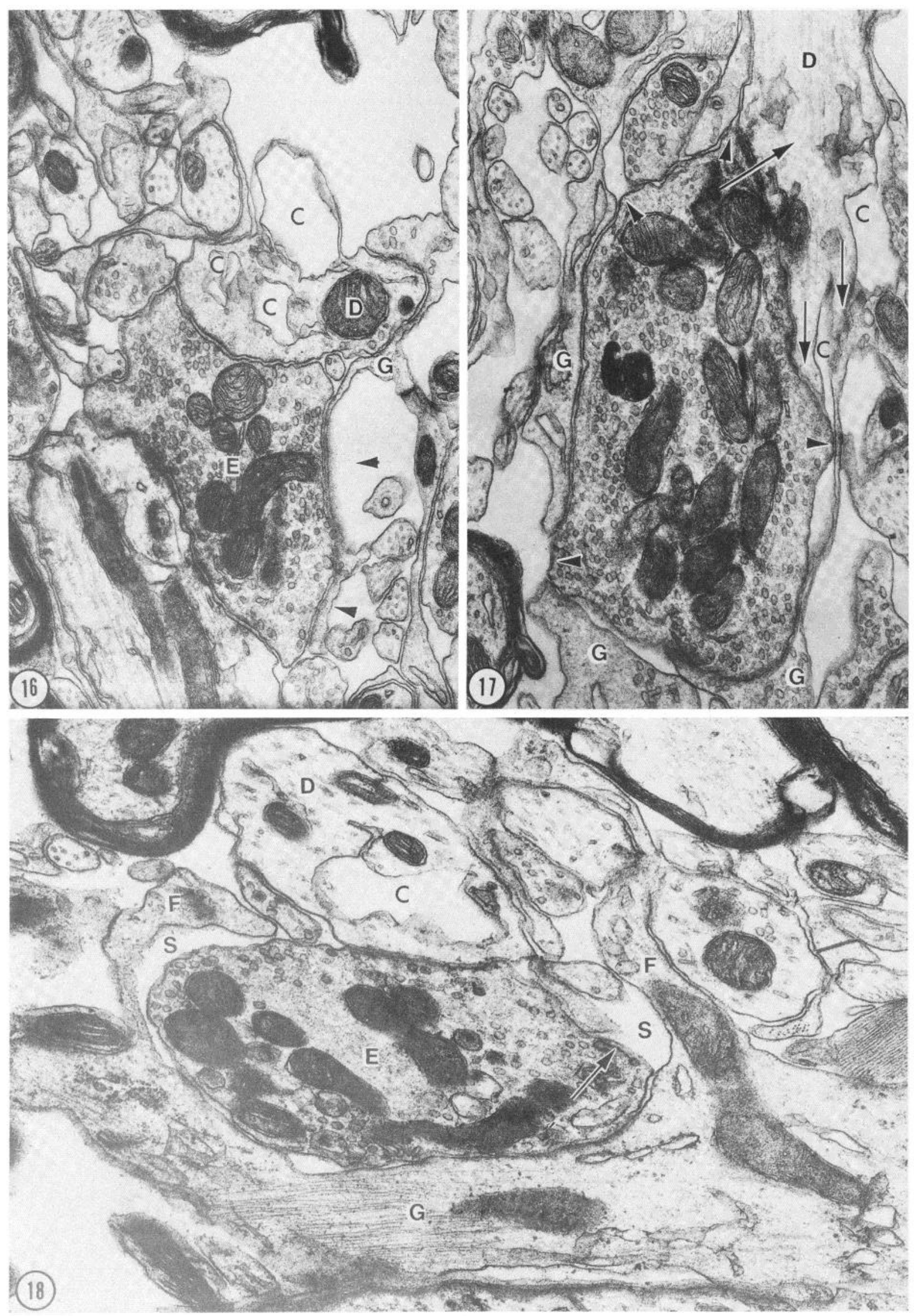
are capable of maintaining their complement of synaptic vesicles for up to 30 days following the injury (Sugimoto and Gobel, 1982). However, two kinds of changes in the properties of injured primary neurons may occur which might directly relate to the cause of the destruction of the small dendrites of secondorder neurons. First, recent studies have shown that some injured primary neurons undergo changes in their electrical properties. For example, some injured primary neurons become spontaneously active, others become hyperactive and exhibit bursting of activity to light mechanical stimulation (Wall and Gutnick, 1974a, b; Govrin-Lippmann and Devor, 1978; Scadding, 1981) while still others become silent (Scadding, 1981). Second, a number of recent studies have suggested that injured primary neurons also undergo a number of internal biochemical changes in which they may fail to maintain production of some of their neurotransmitters as well as their normal complement of transportable proteins (Hall, 1982). For example, substance $P$ has been shown to be lost from the central axonal arbors of peripherally injured primary neurons (Barbut et al., 1981). Fluoride-resistant acid phosphatase has also been shown to be lost from the central axonal arbors of peripherally injured primary trigeminal neurons (Rustioni et al., 1971) and primary spinal neurons (Knyihar and Csillik, 1976). Changes such as these in the normal composition of transportable proteins may alter important sustaining trophic effects by intact primary neurons on the small-caliber dendrites. At this point in time, not enough is known about the properties of injured primary neurons to more precisely link the causative factors of the degenerative dendritic changes in second-order medullary horn neurons to the changes which might have occurred in the injured primary trigeminal neurons.

The destructive effects of peripheral nerve injury may extend beyond the ipsilateral second-order neurons since some cavitated dendrites were also found on the contralateral side in laminae I and II. Studies in which horseradish peroxidase was injected ipsilaterally into the medullary dorsal horn yielded many horseradish peroxidase-filled neurons in the contralateral medullary dorsal horn (Hockfield and Gobel, 1982). These studies suggest that many neurons in the medullary dorsal horn send axon collaterals across the midline to terminate amongst their counterparts on the opposite side. The presence of cavitated dendrites on the side contralateral to the nerve injury may reflect changes in either the electrical or internal biochemical properties in the ipsilateral second-order neurons which could be manifested through their transmedian axon collaterals. Westrum et al. (1976), using silver degeneration stains, also noted degenerative changes on the contralateral side within more rostral parts of the spinal $\mathrm{V}$ nucleus following unilateral peripheral trigeminal nerve injuries. Since recent studies have shown that primary trigeminal axons terminate only ipsilaterally in the trigeminal sensory nuclei (Arvidsson and Gobel, 1981; Westrum et al., 1981), such contralateral degenerative changes might also reflect degenerative changes in the neurons of the contralateral spinal $\mathrm{V}$ nucleus.

Although many small dendrites are destroyed, neurons in laminae I and II do not die as a result of the peripheral nerve injury. Despite the presence of a rare cavity in the cytoplasm of a cell body and the loss of some organelles from some cell bodies (Gobel and Binck, 1977), there are no signs of destruction of neuronal cell bodies or large-caliber dendrites. Large pieces of debris inside of glial processes which might indicate the breakdown of neuronal cell bodies have not been found. Electrophysiological studies following peripheral nerve injuries as a result of either tooth pulp extirpations (Dostrovsky et al., 1982) or transection of the sciatic and saphenous nerve (Devor and Wall, 1978, 1981; Lisney, 1983) have also shown that second-order neurons which normally receive inputs from the injured primary neurons survive. These electrophysiological studies have shown that many of these second-order neurons become spontaneously active and show dramatic changes in their receptive fields. For example, within the first 60 days following tooth pulp extirpations, many neurons in subnucleus oralis which ordinarily have small peripheral receptive fields within a single trigeminal division show enlarged receptive fields which encompass two or even all three trigeminal divisions (Dostrovsky et al., 1982). Neurons in the spinal dorsal horn which ordinarily have receptive fields on the foot after sciatic and saphenous nerve section show receptive fields more proximally on the leg and back (Devor and Wall, 1978, 1981; Lisney, 1983). Since the transfer of inputs from primary neurons to second-order neurons is known to take place on smallcaliber dendrites in the trigeminal sensory nuclei (e.g., see Gobel, 1971, 1974; Westrum and Black, 1971), and in the spinal dorsal horn (Ralston and Ralston, 1979; Gobel et al., 1981a) the changes in receptive fields of these second-order neurons must be a direct result of changes taking place on the smallcaliber dendrites.

In a series of peripheral nerve injury studies in which the superficial radial nerve was transected, cavitation of smallcaliber dendrites in the deeper laminae of the cervical dorsal horn, i.e., in laminae III and IV as well as in laminae I and II occurred during the same 3-month postoperative time period as in the tooth pulp extirpation studies (Sugimoto and Gobel, 1984). This suggests that cavitation of small dendrites and the associated changes in receptive fields may be a stereotypic response of many different kinds of second-order neurons in both spinal and trigeminal systems. The electrophysiological response properties of the different kinds of neurons in laminae I and II in the medullary and spinal dorsal horns, however, remain to be systematically explored following peripheral nerve injury.

Cavitation may be a process by which several kinds of secondorder trigeminal and spinal neurons make alterations in their dendritic arbors throughout their lifetime in response to changes in inputs. In developmental Golgi studies (Falls and Gobel, 1979), many examples of the two major cell types of laminae II, i.e., the islet cells and stalked cells (Gobel, 1978), were found to have many more dendritic branches than the adult forms of these cells. They contained many short branches, and many of these were beaded. In addition to the sheer number of branches, the form of the young dendritic arbors differed markedly from their adult forms. Dendritic branches extended in all directions from many of the cell bodies in almost radial fashion in contrast to the more polarized shapes of the adult

Figure 16. Lamina IIb at 60 days. Two empty scalloped depressions (arrowheads) in the surface of the central axonal ending (E) are indicative of the loss of dendrites from this glomerulus. The axolemma of the scalloped ending in these empty depressions is covered by a fine glial process $(G)$. Small cavities $(C)$ are eroding one of the two remaining dendrites $(D)$ in the glomerulus. Magnification $\times 26,500$.

Figure 17. In this glomerulus, the longitudinally sectioned dendrite $(D)$ at the top, although still connected to the central axonal ending by a junction (oblique arrow), exhibits two long finger-like processes (vertical arrows) which are indicative of previously opened cavities $(C)$. With the exception of a few bare spots, most of the surface of the axolemma is covered by thin glial processes $(G)$. Arrowheads indicate peak-like angulations which reflect traces of the original scalloped contours of the central ending. Magnification $\times 26,600$.

Figure 18. Lamina IIb at 60 days. Indications of severe disruption in this glomerulus include enlarged intercellular space $(S)$ around the central ending $(E)$ and an empty scalloped depression (arrow). The single remaining dendrite $(D)$ abutting the central ending contains a large cavity $(C)$ which is in contact with a cisterna of agranular reticulum. A large glial process at the bottom $(G)$ and its two finger-like extensions $(F)$ partially envelop the central ending. Magnification $\times 38,000$. 
forms of the dendritic arbors. In addition, many immature dendritic arbors contain extremely thick basal dendritic branches, i.e., $>10 \mu \mathrm{m}$ in diameter, which have no counterpart in the adult dendritic arbors. Clearly these cells are drastically modifying the form of their dendritic arbors by eliminating and thinning many branches as they mature. Electron microscopicul examination of these immature dendritic arbors revealed that many of the fine dendritic branches, including the beaded branches, were undergoing cavitation. It was hypothesized that the dendrites of these developing neurons were involuting through the process of cavitation because they failed to establish synaptic connections with primary axonal endings. This hypothesis was proposed since cavitating dendrites were never found in synaptic contact with scalloped primary endings in glomeruli, although they did establish synaptic connections with some dome-shaped nonprimary axonal endings. The present study supports this hypothesis since either the alteration in or interruption of primary inputs consequent to peripheral nerve injury results in the disintegration of some of the fully differentiated adult branches of these cells. As in the developing situation, the presence of synaptic inputs from nonprimary axons is not sufficient to prevent cavitation in the adult dendrites (Figs. 6 and 7).

\section{References}

Arvidsson, J., and S. Gobel (1981) An HRP study of the central projections of primary trigeminal neurons which innervate tooth pulps in the cat. Brain Res. 210: 1-16.

Barbut, D., J. M. Polak, and P. D. Wall (1981) Substance P in spinal cord dorsal horn decreases following peripheral nerve injury. Brain Res. 205: 289-299.

Byers, M. R., S. J. Neuhaus, and J. D. Gehrig (1982) Dental sensory receptor structure in human teeth. Pain 13: 221-235.

Cadden, S. W., S. J. W. Lisney, and B. Matthews (1983) Thresholds to electrical stimulation of nerves in cat canine tooth-pulp with $\mathrm{A} \beta$-, A $\delta$ - and C-fiber conduction velocities. Rrain Res. 261: 31-41.

Corpron, R. E., and J. K. Avery (1973) The ultrastructure of intradental nerves in developing mouse molars. Anat. Rec. 175: 585-606.

Devor, M., and P. D. Wall (1978) Reorganization of spinal cord sensory map after peripheral nerve injury. Nature 275: 75-76.

Devor, M., and P. D. Wall (1981) Effect on peripheral nerve injury on receptive fields of cells in the cat spinal cord. J. Comp. Neurol. 199: $277-291$.

Dostrovsky, J. O., B. J. Sessle, and J. W. Hu (1981) Presynaptic excitability changes produced in brain stem endings of tooth pulp afferents by raphe and other central and peripheral influences. Brain Res. 218: 141-160.

Dostrovsky, J. O., G. J. Ball, J. W. Hu, and B. J. Sessle (1982) Functional changes associated with partial tooth pulp removal in neurons of the trigeminal spinal tract nucleus, and their clinical implications. In Anatomical, Physiological and Pharmacological Aspects of Trigeminal Pain, B. Matthews and R. G. Hill, eds., pp. 293310, Excerpta Medica, Amsterdam.

Falls, W. M., and S. Gobel (1979) Golgi and EM studies of the formation of dendritic and axonal arbors: The interneurons of the substantia gelatinosa of Rolando in newborn kittens. J. Comp. Neurol. 187: 118.

Frank, R. M. (1966) Etude au microscope electronique de l'odontoblaste et du canalicule dentaire humain. Arch Oral Biol. 11: 179-199.

Fried, K., and C. Hilderbrand (1981) Pulpal axons in developing, mature and aging feline permanent incisors. A study by electron microscopy. J. Comp. Neurol. 203: 23-36.

Gobel, S. (1971) Structural organization in the main sensory trigeminal nucleus. In Oral-Facial Sensory and Motor Mechanisms, Y. Kawamura and R. Dubner, eds., pp. 183-204, Appleton-Century-Crofts, New York.

Gobel, S. (1974) Synaptic organization of the substantia gelatinosa glomeruli in the spinal trigeminal nucleus of the adult cat. J. Neu- rocytol. 3: 219-243.

Gobel, S. (1976) Dendroaxonic synapses in the substantia gelatinosa glomeruli of the spinal trigeminal nucleus of the cat. J. Comp. Neurol. 167: 165-176.

Gobel, S. (1978) Golgi studies of the neurons in layer II of the dorsal horn of the medulla (trigeminal nucleus caudalis). J. Comp. Neurol. 180: $395-414$.

Gobel, S., and J. M. Binck (1977) Degenerative changes in primary trigeminal axons and in neurons in nucleus caudalis following tooth pulp extirpations in the cat. Brain Res. 132: 347-354.

Gobel, S., W. M. Falls, and E. Humphrey (1981a) Morphology and synaptic connections of ultrafine primary axons in lamina $I$ of the spinal dorsal horn: Candidates for the terminal axonal arbors of primary neurons with unmyelinated (C) axons. J. Neurosci. 1: 11631179.

Gobel, S., S. Hockfield, and M. A. Ruda (1981b) An anatomical analysis of the similarities between medullary and spinal dorsal horns. In Oral-Facial Sensory and Motor Mechanisms: International Symposium on Oral Physiology, Y. Kawamura and R. Dubner, eds., pp. 211223, Quintessence, Tokyo.

Govrin-Lippmann, R., and M. Devor (1978) Ongoing activity in severed nerves: Source and variation with time. Brain Res. 159: 406-410.

Greenwood, F., H. Horiuchi, and B. Matthews (1972) Electrophysiological evidence on the types of nerve fibers excited by electrical stimulation of teeth with a pulp tester. Arch. Oral Biol. 17: 701-709.

Hall, M. E. (1982) Changes in synthesis of specific proteins in axotomized dorsal root ganglia. Exp. Neurol. 76: 83-93.

Harris, R., and C. J. Griffin (1968) Fine structure of nerve endings in the human dental pulp. Arch. Oral Biol. 13: 773-778.

Hockfield, S., and S. Gobel (1982) An anatomical demonstration of projections to the medullary dorsal horn (trigeminal nucleus caudalis) from rostral trigeminal nuclei and the contralateral caudal medulla. Brain Res. 252: 203-211.

Knyihar, E., and B. Csillik (1976) Effect of peripheral axotomy on the fine structure and histochemistry of the Rolando substance: Degenerative atrophy of central processes of pseudorinipolar cells. Exp. Brain Res. 26: 73-87.

Lisney, S. J. W. (1983) Changes in the somatotopic organization of cat lumbar spinal cord following peripheral nerve transection and regeneration. Brain Res. 259: 31-39.

Ralston, H. J., III, and D. D. Ralston (1979) The distribution of dorsal root axons in laminae I, II and III of the macaque spinal cord: A quantitative electron microscope study. J. Comp. Neurol. 1: 643-684.

Ruda, M. A., and S. Gobel (1980) Ultrastructural characterization of axonal endings in the substantia gelatinosa which take up $\left[{ }^{3} \mathrm{H}\right]$ serotonin. Brain Res. 184: 57-83.

Rustioni, A., S. Sanyal, and H. G. J. M. Kuypers (1971) A histochemical study of the distribution of the trigeminal divisions in the substantia gelatinosa of the rat. Brain Res. 32: 45-52.

Scadding, J. W. (1981) Development of ongoing activity, mechanosensitivity, and adrenalin sensitivity in severed peripheral nerve axons. Exp. Neurol. 73: 345-364.

Sugimoto, T., and S. Gobel (1982) Primary neurons maintain their central axonal arbors in the spinal dorsal horn following peripheral nerve injury: An anatomical analysis using transganglionic transport of horseradish peroxidase. Brain Res. 248: 377-381.

Sugimoto, T., and S. Gobel (1984) Dendritic changes in spinal dorsal horn following transection of a peripheral nerve. Brain Res., in press.

Wall, P. D., and M. Gutnick (1974a) Properties of afferent nerve impluses originating from a neuroma. Nature 248: 740-743.

Wall, P. D., and M. Gutnick (1974b) Ongoing activity in peripheral nerves: The physiology and pharmacology of impulses originating from a neuroma. Exp. Neurol. 43: 580-593.

Westrum, L. E., and R. G. Black (1971) Fine structural aspects of the synaptic organization of the spinal trigeminal nucleus (pars interpolaris) of the cat. Brain Res. 25: 265-287.

Westrum, L. E., R. C. Canfield, and R. G. Black (1976) Transganglionic degeneration in the spinal trigeminal nucleus following removal of tooth pulps in adult cats. Brain Res. 101: 137-140.

Westrum, L. E., R. C. Canfield, and T. A. O'Connor (1981) Each canine tooth projects to all brain stem trigeminal nuclei in cat. Fxp. Neurol. 74: 787-799. 